\title{
AUTOMATED LONG-TERM POLYSOMNOGRAPHY ANALYSIS WITH WAVELET PROCESSING AND ADAPTIVE FUZZY CLUSTERING
}

\author{
Chin-Feng ChaO ${ }^{1}$, Joe-Air JiAng ${ }^{1}$, Ming-Jang ChiU ${ }^{2}$, Ren-Guey LeE ${ }^{3}$ \\ ${ }^{1}$ Department of Bio-Industrial Mechatronics Engineering, \\ National Taiwan University, Taipei, Taiwan \\ ${ }^{2}$ Department of Neurology, National Taiwan University Hospital, Taipei, Taiwan \\ ${ }^{3}$ Department of Electronic Engineering, National Taipei University of Technology, Taipei, Taiwan
}

\begin{abstract}
To assist in the inspection of sleep-related diagnosis and research, an adaptive method for processing long-term polysomnography (PSG) is proposed in this paper. The extracted features of segmented PSG based on wavelet analysis can be used for clustering the segments with similar pattern into a group. The adaptive fuzzy clustering was used to estimate the clusters within the PSG recordings, the optimal number of clusters and the optimal features of an individual subject. The novel method with the adaptive-to-subject concept exhibits four advantages in comparison with other approaches: (1) Full automated, (2) adaptive to the diversity of physiological signals among subjects, (3) less sensitive to noise and artifacts, (4) effective visualization of analysis results for clinicians. The simulation results show the superiority of the proposed method in long-term PSG analysis.
\end{abstract}

Biomed Eng Appl Basis Comm, 2006(June); 18: 119-123.

Keywords: polysomnography; wavelet transform; fuzzy clustering

\section{INTRODUCTION}

The polysomnography (PSG) is a set of measurements that includes electroencephalogram (EEG), electromyogram (EMG), electro-oculogram (EOG), and other signal types for clinical medicine. The recognition of PSG into the different sleep states gives significant indications for the diagnosis and treatment for patients for sleep-related disorders [1]. Such applications normally required large quantities of

Received: Jan. 5, 2006; Accepted: March 27, 2006 Correspondence: Ren-Guey Lee, Professor Department of Electronic Engineering, National Taipei University of Technology, Taipei, 10643 Taiwan E-mail: evans@ntut.edu.tw prolonged PSG recordings, with length of more than several hours or even a day. Furthermore, interpretation and managing essential variation of physiological events by long-term PSG was a very tedious and time-consuming operation. To improve the efficiency of PSG inspection, the need of automatic processing and analysis is all too obvious in practice.

To date, many automatic methods have been proposed for PSG processing. Most of these analysis methods tried to imitate visual inspection according to some standards, such as $\mathrm{R}$ and $\mathrm{K}$ rules [2]. The rule-based logics were used to deal with the extracted features of PSG [3-4]. However, these methods suffered a critical problem, that is, the great diversity of biological activities among subjects. The pre-defined rules or threshold settings for the presence 
of some specific activities were most based on the empirical observations of clinicians [1]. The rule-based settings might be suitable for reviewing/diagnosis for clinicians, but may not be reliable for a robust/adaptive principle of accurate computer analysis. Recently, Agrawal and Gotman introduced a self-organization method based on k-means to cluster segments of all night PSG for sleep staging [4-5]. This idea reduced the influence of the above-mentioned problems but there were still some problems of the methods: (1) the $k$-means clustering was known to be very sensitive to noise [6]; (2) the number of clusters of PSG recordings still needed to be defined by users: The problem is that mostly we were incapable to know the true number of clusters in a recording; (3) the fixed feature set was used for each subject: the analysis may not be reliable for every subject.

Since the physiological activities are quite different between subjects, it can not reach optimal results for every distinct subject by extracting a fixed feature set for clustering/classifying the different patterns of bio-signals. In general, feature selection was an essential procedure in supervised learning used to minimize the classification error, but for unsupervised clustering, it was rarely mentioned in the literatures [7]. With a well-chosen feature set, the effect of noisy sample can be reduced and all the samples could be separated clearly. On the contrary, with the poorly-chosen feature set, the samples would hard to be differentiated in the feature space [7]. Therefore, an adaptive-to-subjects method for clustering long-term PSG with noise needs to be developed.

To the best of our knowledge, the present study is the first attempt at applying adaptive-to-subject techniques to process the features of bio-signals and cluster the segments from a long-term PSG recording. To overcome the problems and achieve an optimal clustering result, the proposed system integrated several superior methods for signal processing, segmentation, feature subset searching, clustering, and validity measure into the overall wavelet-based analysis and adaptive fuzzy clustering algorithms. The illustrated in Fig. 1 and described in following sections.

The remainder of this paper is organized as follows. The procedure of the proposed method is developed in Section 2. Experimental results are shown in Section 3. Finally, some conclusions are given in Section 4.

\section{METHOD}

\subsection{Subjects and Recordings}

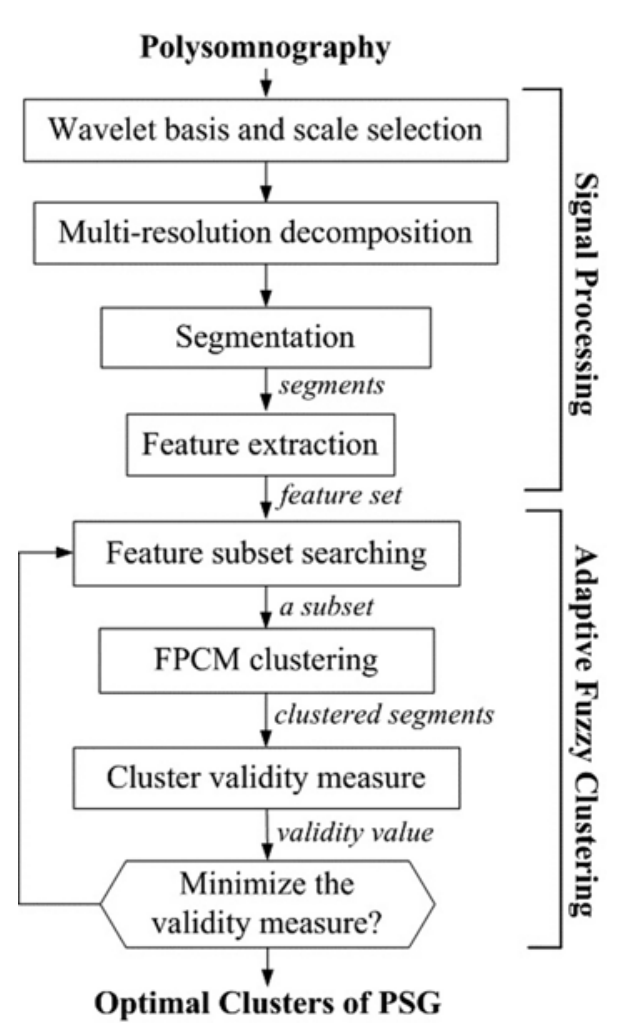

Fig. 1. The structure of automated PSG analysis.

The PSG recording for simulation was obtained from the Sleep-EDF Database in PhysioBank, numbered st $2022 \mathrm{j} 0$ [8]. The recording was acquired from a 35-year-old woman who had mild difficulty falling asleep but was otherwise healthy. Four channels, horizontal EOG, FpzCz, PzOz EEG and submental EMG, were sampled at $100 \mathrm{~Hz}$ with length of about 8 hours.

\subsection{Wavelet Multi-resolution Analysis}

There were a large number of comprehensive applications based on wavelet transforms for biomedical engineering [9]. It contained filtering, detection, feature extraction and modeling for biosignal analysis. In many applications, the discrete wavelet transform (DWT) is sufficient to satisfy the requirements of analysis. The DWT is dyadic WT when the scaling parameter, $a$, is a number with power of two, i.e. $a=2^{j}, j \in Z$. Mallat's fast algorithm for DWT is computed as follows [10]:

$$
A_{j}(n)=\sum_{k=-\infty}^{+\infty} g(k-2 n) A_{j-1}(k)
$$

$$
D_{j}(n)=\sum_{k=-\infty}^{+\infty} h(k-2 n) A_{j-1}(k)
$$


where $A_{j}$ is called the $j$ th approximation, or approximation at level $j ; D_{j}$ is called the $j$ th detail, or detail at level $j$. The filters, $h$ and $g$, used to decompose a signal are known as wavelet filters and scaling filters, respectively.

Two primary rhythms alpha, beta and slow-wave activity of EEG, were extracted by the selected basis Symlet-8 without suffering time-shift [10], as shown in Fig. 2. Since the WT is somehow a correlation measure between the signal and the wavelet basis, to select a temporal pattern similar to EEG rhythms, such as Symlet-8, can be capable to extract the dynamic variations completely. The alpha, beta rhythms and slow-wave activity were extracted by Symlet- 8 as the $2^{\text {nd }}, 3^{\text {rd }}$ details and $3^{\text {rd }}$ approximation, respectively. Furthermore, the sleep spindles were extracted by Morlet basis which has similar pattern to spindles, and then an empirical threshold for sleep spindle was used to achieve the detections [11].

\section{Step1: Segmentation}

Frequency-weighted energy (FWE), defined by the nonlinear energy operator (NLEO) [5], which is sensitive to the variation of energy and frequency in a signal. We use this idea to simultaneously estimate segment boundaries of long-term PSG recordings and divided them into quasi-stationary segments. After segmentation, the activity of signals tended to be similar within a segment, and different between segments. A segmentation criterion is generated for the two EEG and the EMG channels as,

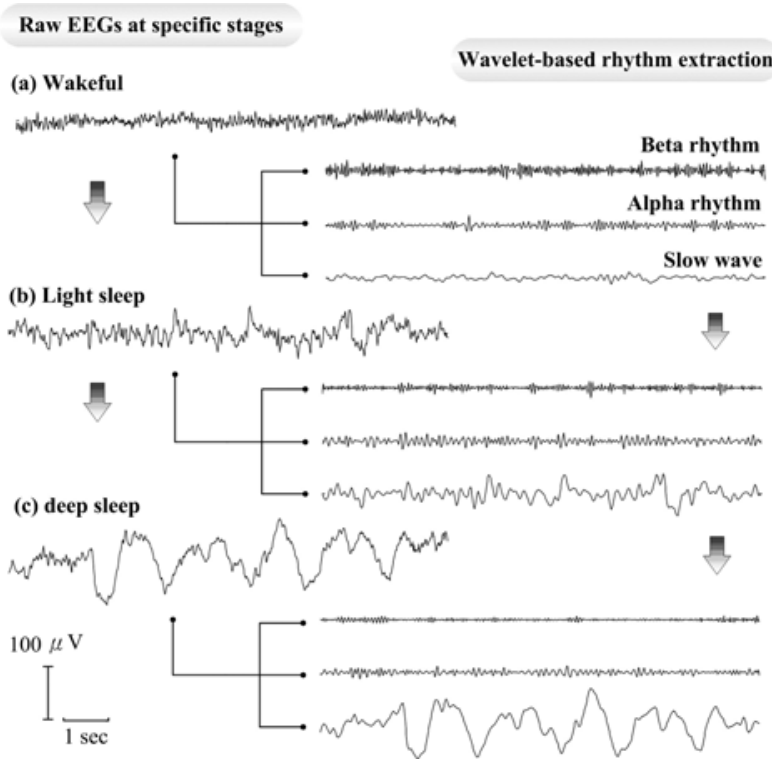

$$
\begin{gathered}
G_{N L E O}(n)=\left|\sum_{m=n-N+1}^{n} E(m)-\sum_{m=n+1}^{n+N} E(m)\right| \\
E(n)=x(n-1) x(n-2)-x(n) x(n-3)
\end{gathered}
$$

where $2 N$ is the sliding window size. The segmentation criteria are linearly summed to obtain the overall segmentation criterion [5].

\section{Step2: Segment feature extraction}

The feature vector describing sleep-related attributes are extracted in each segment by wavelet analysis. The following features represent each segment of the PSG [4]: amplitude and frequencyweighed energy (FWE) of three primary EEG rhythms and the EMG channel, presence of spindles in the frontal EEG channel, alpha-slow-wave index (ASI) and theta-slow-wave index (TSI) for the two EEG channels, and presence of eye-movement index (EMs) in the EOGs. Thus, each segment is parameterized by a 14-dimensional feature vector.

\subsection{Adaptive Fuzzy Clustering}

Suppose that there were $N$ segments in a PSG recording, and given a set of 14 extracted features in section 2.1. The proposed method can be adapted to subjects by selecting a feature subset of size $m$ and estimating the number of clusters among $N$ segments that leads to the best clustering result for each subject.

\section{Step 1: Feature subset searching}

Concerning the computational complexity and the optimality, we chose the Sequential Forward Floating Search (SFFS) method to generate feature subsets [7]. The effective method used "Plus $l$ - take away $r$ (features)" strategy and the values of $l$ and $r$ are determined automatically and updated dynamically. It provides close to optimal solution almost as well as branch-and-bound algorithm at a much lower computational cost.

\section{Step 2: FPCM clustering}

The generated subset in the previous step was then fed into FPCM. FPCM was a robust clustering method that hybridizes the Fuzzy-C-means and Possibilistic-CMeans models, enjoys their benefits and eliminates the problems of them [6]. Since the membership of FPCM was not restricted to 1 , the membership of noisy segments would be relatively lower than other segments. That is, the influence of noise and outliers was reduced. In order to find optimal number of clusters in the segment feature space, the number of clusters was set to $\mathrm{c}=2 \sim 8$ for iterative operation.

Fig. 2. Wavelet-based EEG rhythms extraction. 


\section{Step 3: Subset evaluation by cluster validity measure}

After iterative clustering procedures, the validity measure in this investigation was to estimate the "Goodness" of clusters by compactness and scatter separability of the clustered data. Many methods of validity measure have been proposed in the literatures, but some were effected critically by the noisy data and the poorly separated clusters. In practice, since physiological signals were easily contaminated by artifacts, the noisy data was existed in the extracted feature space. Therefore, we adopted a more reliable validity function, $V_{W J S}$, to avoid the above-mentioned problems [12].

Repeat Step 1 3 until the search completes or minimizing the estimated validity measure. Finally, the optimal subset and the number of clusters can be obtained. The output of adaptive fuzzy clustering is the cluster centers and cluster labels for each segment.

\section{Step 4: Visualization}

To display the large quantity of PSG analysis results consisted of segment lengths/boundaries, selected feature subset, segment clusters for clinicians is an essential work in clinical practice. The effective and clear presentation can further improve the efficiency of PSG inspection [5]. Therefore, the analysis results were presented in a compressed illustration form. The segments were merged into 30sec segments (epochs) as a representative unit for the standard visually inspection. The merged epochs belonged to which cluster depended on which segment cluster occupies the largest fraction of the epoch.

\section{RESULT}

The simulation results of 8-hour PSG recordings are illustrated in Fig. 3. The selected feature subset for this subject by the proposed algorithm consisted of ASI, EMs energy of EMG and beta activity. The optimal number of clusters in this case was estimated as five. The colored signals in the five blocks are respectively the representative patterns for five clusters in the PSG recording. Comparatively, the signals have similar patterns within a cluster, but different between clusters. The chronological sequence (temporal profiles) that has been color coded to relate each 30sec segment to a specific cluster. The duration of each segment in the sequence is proportional to the actual duration of PSG segments.

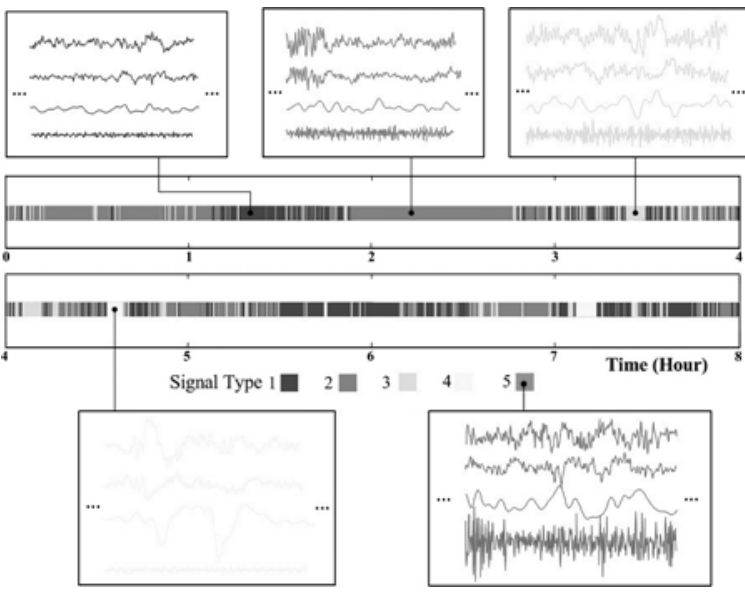

Fig. 3. Representative pattern of clusters and chronological sequence of an 8-hour PSG recording.

\section{CONCLUSION}

This paper proposed an adaptive method for processing long-term polysomnography to assist in the inspection of sleep-related diagnosis and research. The extracted features of segmented PSG based on wavelet analysis can be used for clustering the segments with similar pattern into a group. The adaptive fuzzy clustering was used to estimate the clusters within the PSG recordings, the optimal number of clusters and the optimal features of an individual subject. The novel method with the adaptive-to-subject concept exhibits four advantages in comparison with other approaches: (1) Full automated, (2) adaptive to the diversity of physiological signals among subjects, (3) less sensitive to noise and artifacts, (4) effective visualization of analysis results for clinicians. The simulation results show the superiority of the proposed method in longterm PSG analysis.

According to Fig. 3 and the $\mathrm{R}$ and $\mathrm{K}$ rules [2], the signals of the blue and red clusters occupying the most portion of the temporal sequence can be inspected as the activities of the light sleep. Therefore, based on the results, the subject was known to be most in the condition of light sleep. The other clusters represent the wakeful state, rapid- eye-movement sleep and artifact contaminated were colored as green, yellow and purple in the sequence, respectively. The appearance of deep-sleep activity was few in this case and insufficient to form an individual cluster. Therefore, the results just match the sleep problems of subjects described in section 2.1. From the illustration, the physiological activity over several hours of a subject can be reviewed obviously and efficiently. 
The proposed method developed by wavelet-based processing and adaptive fuzzy clustering can be adaptive to the diversity of subjects with an accurate analysis. We believe that the advantages of each procedure make it more reliable and can be used in practice with an effective visualization for clinical usage.

\section{REFERENCE}

1. Thomas $P$ and Regina C: Computer based sleep recording and analysis. Sleep Medicine Reviews 2000; 4: 131- 148 .

2. Rechtschaffen A and Kales A-Eds: A manual of standardized terminology. in Techniques and Scoring System for Sleep Stage of Human Subjects, 1968.

3. Kemp B: A proposal for computer-based sleep/wake analysis - consensus report. J. Sleep Res. 1993: 179-185.

4. Agarwal R and Gotman J: Computer- assisted sleep staging. IEEE Trans. Biomed. Eng. 2001; 48: 14121423.

5. Agarwal R and Gotman J: Automatic EEG analysis during long-term monitoring in ICU. Electroencephalo- graphy and Clinical Neurophysiology 1998; 107: 44-58.

6. Pal NR, Pal K, Keller JM, and Bezdek JC: A possibilistic fuzzy C-Means clustering algorithm. IEEE Trans. Fuzzy Syst. 2005; 13: 517-529.

7. Jain AK, Duin RPW, and Mao J: Statistical pattern recognition-a review. IEEE Trans. Pattern Anal. Machine Intell. 2000; 22: 4-27.

8. Physionet, http://www.physionet.org/

9. Unser $M$ and Aldroubi A: A review of wavelets in biomedical applications. Proc. IEEE 1996; 84: 626638.

10. Percival DB and Walden AT: Wavelet methods for time series analysis, 1st ed., 40 West 20th street, New York, Cambridge University Press, 2000: 115121.

11. Schimicek P, Zeitlhofer J, Anderer P, and Saletu B: Automatic sleep spindle detection procedureaspects of reliability and validity. Clinical Electroencephalography 1994; 25: 26-29.

12. Sun H, Shengrui W, and Jiang Q: FCM-based model selection algorithm for determining the number of clusters. Pattern Recognition 2004; 37 : 2027-2037. 